\title{
Why Academics Choose to Publish in a Mega-Journal
}

\author{
Jovan Shopovski $^{1} \&$ Dejan Marolov ${ }^{1}$ \\ ${ }^{1}$ European Scientific Institute (ESI) \\ Correspondence: Jovan Shopovski, European Scientific Institute (ESI). E-mail: jovanpraven@yahoo.com
}

Received: August 16, 2017

Accepted: August 31, $2017 \quad$ Online Published: September 4, 2017

doi:10.5539/jel.v6n4p348

URL: http://doi.org/10.5539/jel.v6n4p348

\begin{abstract}
With their broad scope, high publishing volume, a peer review process based on the scientific soundness of the content, and an open access model, mega journals have become an important part of scholarly publishing.

The main aim of this paper is to determine the most important factor that influenced researchers' decisions to submit their academic work to these type of journal. To this end, an online survey has been disseminated from November 2016 to August 2017, targeting the corresponding authors of the European Scientific Journal, ESJ. Data from 413 corresponding authors was collected.

The focus was mainly on how they discover the journal and what led them to submit a paper to the journal. However, questions concerning their satisfaction with the peer review procedure were also part of the survey.

The results have shown that a recommendation of a colleague is not only the main channel through which authors found out about the journal, but is also the major reason they decided to submit their paper to a mega-journal. Furthermore, the quality of the editorial board of the journal, the strong portfolio of papers and the open access concept are also significant factors in encouraging submission to a mega-journal. A majority of the respondents are satisfied with the communication and peer review procedure of the mega-journal, which might encourage new submissions in the future.
\end{abstract}

Keywords: academic publishing, mega-journal, open access

\section{Introduction}

Open-access mega-journals are one of the innovations that evolved with the promotion of the open-access concept and its goal to make scholarly publications freely available.

It is difficult to establish a precise definition of the term "mega-journal". However, there are four major characteristics a journal should meet in order to be recognized as a mega-journal: 1) High publishing volume, 2) Broad scope, 3) Open-access publishing model, and 4) Objective peer review based on scientific soundness of the content (rather than novelty or potential impact) (Wakeling et al., 2017). Bienfield (2013) categorizes these four characteristics as primary, while listing some other as secondary. A mega-journal has to meet all of the primary elements and most of the secondary such as moderate article processing charges, using Almetrics, rapid peer review, using academic editors, etc. (Bjork, 2015). Nevertheless, there are still many relative terms presented in the area, such as high publishing volume, moderate article charges or reputable publisher (Spezi et al., 2017).

Even though a relatively new publishing model, invented in the last decade, mega-journals have become an important channel for disseminating academic research (Solomon, 2014). Nowadays, apart from newcomers, there are also examples of established journals transitioning from the traditional publishing model to the open access mega-journal model (Wakeling et al., 2017).

Furthermore, there is insufficient independent data and analysis to fully investigate the phenomenon of mega journals. The present studies are mainly focused on areas closely related to the mega-journals, such as open access, the value of peer review but not dealing specifically with the mega-journals per se (Spezi et al., 2017).

Although some publishers have conducted author surveys, there are low numbers of independent surveys of mega-journal contributors. Issues such as the experience of the authors with the submission, peer review and publication processes are very important. Moreover, revealing the factors that influence authors who choose to publish in a mega-journal are certainly important in order to understand this new model of academic publishing (Solomon, 2014). 
To that end, we conducted a survey that targets the corresponding authors of one of the mega-journals. It is hoped that this will enrich the academic literature and create an understanding of how authors come to know about the mega-journals, the most important factor that prompts them to submit their paper to the journal, as well as their satisfaction with the peer review procedure and potential return rate, which may encourage the progress and further development of the mega-journals.

\section{Methodology}

\subsection{Sample}

We have decided to select the European Scientific Journal, ESJ mainly because we are involved in and closely familiar with its publication practices and procedures. The peer review procedure of this journal is beyond question. Every single paper submitted to the journal undergoes a formal peer review procedure. More than one reviewer examines most of the papers. Moreover, ESJ publishes a high number of papers in every academic discipline, promoting the gold open-access model. Finally, the recent metrics of Google Scholar have also revealed a considerable citation impact and progress of the journal (Note 1).

The sample for the survey consisted of corresponding authors who successfully published their papers in the European Scientific Journal, ESJ. The main focus was on those who published their papers in the period when the survey was active, i.e., from November 2016 to August 2017. However, dissemination of the survey to the authors was through the journal's official email address and was available during the communication with other authors as well. To that end, the exact response rate cannot be determined, neither was the exact year of authors' publications checked. In any case, there were no substantial changes in the ESJ's peer review procedure, neither has the amount of the publications fee been changed. However, there has been progress in the list of editorial members, the number of articles over the years and the visibility of the journal.

\subsection{Survey}

In order to maximize the number of responses we have rationalized the design of the survey. General data were required from the respondents such as name, surname and university affiliation. These data were obligatory and we were familiar with the identity of the respondents. Moreover, the second question in the survey allowed the respondents to pick one of the options on how they found out about the European Scientific Journal, ESJ. The third question and a main goal of the study, was to disclose the principal reason they decided to submit their paper to the journal. In both questions, several options were offered with only one to be chosen. A graph category "other" was also left in the end. Multiple answers were not allowed in order to keep the focus on the main factor that influenced the decision to submit a paper to this mega-journal.

In addition, the authors were asked to evaluate their communication with the editorial office and their satisfaction with the journal's peer review procedure.

Reminder to the authors to complete the survey was not sent.

In order to collect the answers, Survey Monkey software was used. The link between the survey and the invitation was included in the announcement sent to the authors, as a notification that their paper had been successfully published. This highlighted that completing the survey would take 8 minutes of their time.

Responses from 413 ESJ's corresponding authors were collected.

\section{Results}

The ESJ editorial office for the purpose of the study provided the contacts of the corresponding authors. They were contacted directly through their email addresses. Moreover, a link to the survey, with an invitation, was available in the acceptance letter, sent by the editorial office.

As mentioned earlier, at the beginning of the survey authors were asked to reveal their personal data such as name, surname, university and email address, which was mandatory.

Furthermore, they were asked how they came to know about the European Scientific Journal, ESJ. The intention was to collect data and identify the most usual way through which authors find out about the mega-journal and potentially to reveal how mega-journals have become a popular choice for scholarly publication, as they are nowadays.

Even though this question was not mandatory, only one of the respondents failed to answer it. Undoubtedly, the recommendation of a colleague is the most common way through which authors discover the mega-journal. In the case of the European Scientific Journal, ESJ. 64.56\% (266) of the respondents selected this response. 
However, a significant fraction of the responses went to Google and Google Scholar-about 25\% (79 and 24) in total. Finally several authors responded that they found out about the journal through social media or chose the option "other".

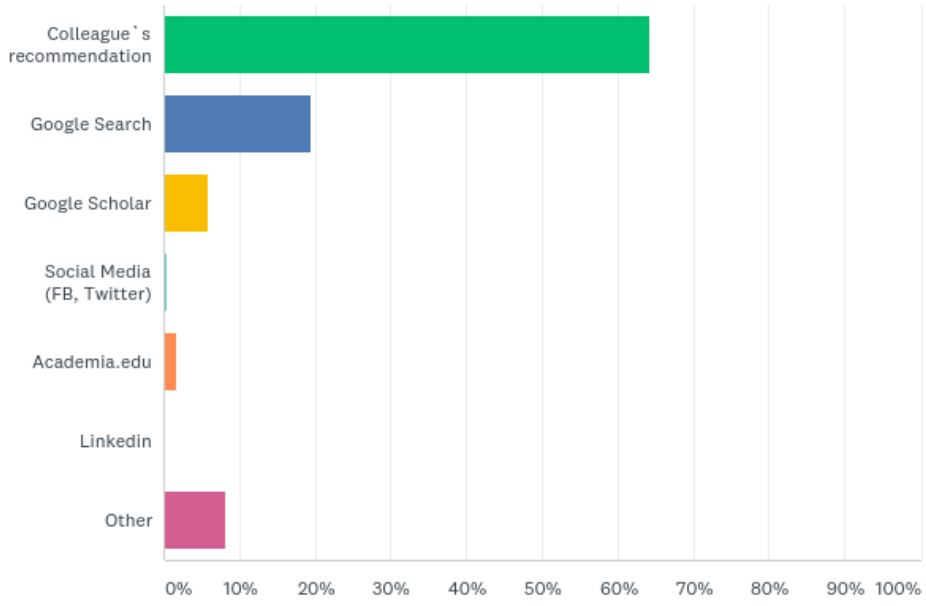

Figure 1. What made you to submit your paper to ESJ?

Source: Survey.

Authors' decisions about whether to submit their academic articles to a mega-journal are influenced by multiple factors. Furthermore, a previous study has confirmed that rapid peer review, open access, journal impact and quality are considered significant factors that trigger submission to mega-journals. However, some other factors, such as a journal's broad scope, the amount of publication fee and recommendation of a colleague also influence the decision-making process (Solomon, 2014).

To that end, this study was designed to collect data regarding the single most important factor, which plays a major role in encouraging authors to submit their papers to a mega-journal. Thus, the authors were obliged to choose only one of the options that made them to submit their paper to the European Scientific Journal, ESJ. However, there was an option "other" in the end, where they could add new options as well as their own comments.

Since a significant part of the corresponding authors was submitting their papers to ESJ for a first time, it was clear that they would not have prior experience with the approximate duration of the review process. Therefore, "agility" instead of "agile peer review procedure" was included as an option. However in July 2017, "agility" was changed to "agile peer review procedure" and one new option "amount of the publication fee" was added.

The options given were: 1) Colleague's recommendation, 2) Quality editorial board, 3) Good archive (papers), 4) Open access, 5) Visibility, 6) Agility (latter changed to Agile peer review procedure), 7) Amount of the publication fee (added as from July), and 8) Other.

It is a well-known phenomenon, even in the case of long-established publishers, that many authors are frustrated with the time that the peer review procedure takes (Cressey, 2015). Therefore we decided to clarify the option "agility" by correcting it to "Agile peer review procedure". The amount of the publication fee was also added as one of the options. However, there were no changes in the results after these corrections.

It turned out that the recommendation of a colleague is the most significant factor that leads authors to submit their paper to a mega-journal. $137(33.25 \%)$ of the respondents indicated that the good recommendation of their colleagues had a major influence on their decision to submit the paper to ESJ. For $102(24.76 \%)$ of the respondents, the quality editorial board plays the main role in their choice. The open access model with 64 $(15.53 \%)$ is the third factor that influenced authors the most. Agility/Agile peer review procedure with 42 $(10.12 \%)$ responses and good archive (papers) with $33(8 \%)$ are less significant factors. The amount of the publication fee from the moment it was included received 5\% (2 votes out of 40$)$ and visibility $(0.74 \%)$ are at the end of the list. 


\section{Q3 What made you to submit your paper to ESJ?}

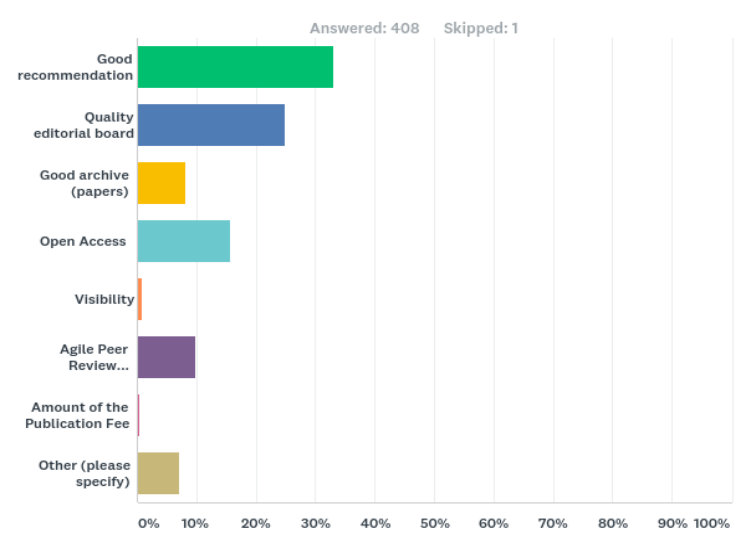

Figure 2. What made you to submit your paper to the European Scientific Journal, ESJ

Source: Survey.

$7 \%$ (29) of the respondents selected the option "other". Mainly the comments in this section were combinations of several factors or indicated the agile peer review procedure as the most important factor.

Furthermore, the corresponding authors were asked about their communication with the ESJ editorial office and whether they were satisfied with the ESJ peer review procedure. The aim of these questions was to determine the satisfaction with the process and potential new submission to the journal.

\begin{tabular}{c|lc}
\hline Answer Choices & Responses & 307 \\
\hline Excellent & $74.51 \%$ & 100 \\
\hline Good & $24.27 \%$ & 4 \\
\hline Average & $0.97 \%$ & 1 \\
\hline Bad & $0.24 \%$ & 412 \\
\hline TOTAL & & 4 \\
\hline
\end{tabular}

Figure 3. How was your communication with the ESJ editorial office

Source: Survey.

It is clear that more than $2 / 3$ of the authors are highly satisfied with the communication with the journal, rating it as an excellent one. The next largest number describe it as a good one. Four respondents rated the communication as average and only one as bad. 


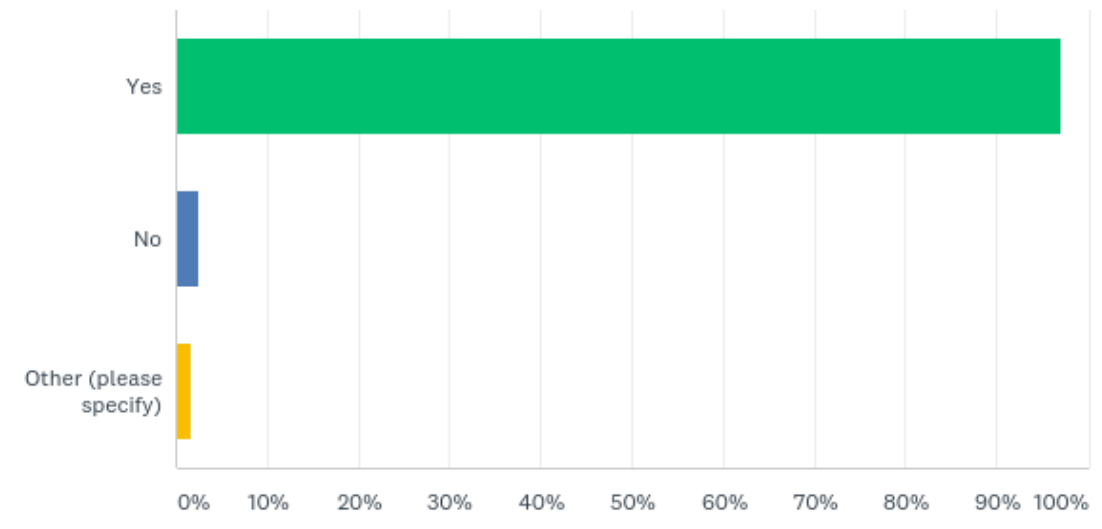

Figure 4. Are you satisfied with the ESJ peer review procedure

Source: Survey.

394 (97\%) people indicated satisfaction with the ESJ peer review procedure by providing an affirmative answer to this question. $10(2.5 \%)$ people answered negatively and $7(1.7 \%)$ chose the option "other" with their comment in addition.

\section{Discussion}

Mega-journals are becoming an important part of scholarly publishing. A broad range of academics choose to publish their academic work in this kind of publications (Solomon, 2014). Therefore it could be of great importance to examine authors' preferences and their experience with the mega-journals. This study should enrich the literature concerning mega-journals through examining the connections between academic authors and these types of journals.

The study has several limitations. Firstly, the opinions of the corresponding authors of only one mega-journal are taken into consideration. This may not represent a general status of mega-journals. Furthermore, in order to increase the response rate the survey was kept short. Therefore it lacks thorough information.

There is no classification by countries, neither a comparison to determine if authors from specific countries demonstrate similarities in their responses. The study also did not examine which portion of the submissions was previously submitted to other journals (re-submissions). Finally, the survey was not anonymous. Therefore authors may not have been freely critical towards the ESJ peer review procedure and/or their communications with the editorial office, especially if they are planning new submissions with the journal.

The European Scientific Journal, ESJ attracts submissions from all around the globe. The submitted papers are in various academic subjects from social sciences through humanities to life sciences. Most of the corresponding authors are academics since a university is called for in their affiliation field.

Recommendation of a colleague turned to be not only how the authors found out about the mega-journal but also the major factor that influenced their decision to submit their paper. It can be said, that when it comes to choosing a medium for publishing a paper, discussion and consultations among colleagues is widely represented. Academics highly value the experience and recommendations of their colleagues.

Moreover, many authors especially value the quality of the editorial board of the European Scientific Journal, ESJ. It is a long list of reviewers from all around the globe, covering various academic disciplines. It could refer to the reliability of the journal and its quality.

Finally, very encouraging news for the open access model and its advocates is the fact that academics appreciate the openness of their papers. A significant number of authors selected open access as the main reason they submit their paper to the mega-journal. This is certainly an area with a progressive future. 


\section{References}

Binfield, P. (2013). Open access megajournals-Have they changed everything? Creative Commons. Retrieved from http://creativecommons.org.nz/2013/10/open-access-megajournals-have-they-changed-everything/

Bjork, B.-C., \& Solomon, D. J. (2014). Developing an effective market of open access article processing charges. Final report to the Wellcome Trust. Retrieved from http://www.wellcome.ac.uk/About-us/Policy/Spotlight-issues/Open-access/Guides/WTP054773.htm

Cressey, D. (2015). Concern raised over payment for fast-track peer review. Nature. https://doi.org/10.1038/nature.2015.17204

Shin, E.-J. (2017). Can the Growth of Mega-Journals Affect Authors' Choice of Journal? Serials Review, 43(2), 137-146. https://doi.org/10.1080/00987913.2017.1313092

Solomon, D. J. (2014). A survey of authors publishing in four mega journals. Peer J, 2, e365. https://doi.org/10.7717/peerj.365

Spezi, V., Wakeling, S., Pinfield, S., Creaser, C., Fry, J., \& Willett, P. (2017). Open-access mega-journals: The future of scholarly communication or academic dumping ground? A review. Journal of Documentation, 73(2), 263-283. https://doi.org/10.1108/JD-06-2016-0082

Wakeling, S., Willett, P., Creaser, C., Fry, J., Pinfield, S., \& Spezi, V. (2017). Transitioning from a Conventional to a "Mega". Medicine.

Note

Note 1. ESJ $\mathrm{h}$ index is 26 and $\mathrm{h}$ median 32 . Available at https://scholar.google.com/citations?hl=en\&view_op=list_hcore\&venue=MQTZLImPvHEJ.2017.

\section{Competing Interests}

The authors are part of the editorial office/editorial board of the European Scientific Journal, ESJ.

\section{Copyrights}

Copyright for this article is retained by the author(s), with first publication rights granted to the journal.

This is an open-access article distributed under the terms and conditions of the Creative Commons Attribution license (http://creativecommons.org/licenses/by/4.0/). 